\title{
Enhancement of Sister Chromatid Exchanges (SCEs) in Peripheral Blood Lymphocytes of Women with Polycystic Ovary Syndrome (PCOS) in Vitro
}

\author{
Evagelia Dafa1, Maria Kontou1, Tzegiaver B. Mantratzi², Emmanouel N. Kontomanolis², \\ Nikoletta G. Koutlaki ${ }^{1}$, Vasilios A. Liberis ${ }^{1}$, Theodore S. Lialiaris ${ }^{{ }^{*}}$ \\ ${ }^{1}$ Laboratory of Genetics, Medical School, Democritus University of Thrace, Alexandroupolis, Greece \\ ${ }^{2}$ Department of Obstetrics and Gynaecology, Medical School, Democritus University of Thrace, Alexandroupolis, \\ Greece \\ Email:
}

Received 22 June 2015; accepted 11 July 2015; published 15 July 2015

Copyright (C) 2015 by authors and Scientific Research Publishing Inc.

This work is licensed under the Creative Commons Attribution International License (CC BY).

http://creativecommons.org/licenses/by/4.0/

(c) $\underset{\mathrm{EY}}{\mathrm{B}}$ Open Access

\section{Abstract}

The aim of the present study was to determine the level of underlying DNA damage in females with PCOS. Twenty-two women with PCOS and twenty-two healthy controls were included in this study. Patients were further categorized into three phenotypic subgroups: Subgroup A, oligo/anovulation (ANOV) and polycystic ovaries (PCO); Subgroup B, hyperandrogenism (HA) as a main characteristic (HA + ANOV or HA + PCO); Subgroup $C$, all three conditions present (HA + ANOV + PCO). The frequency of sister chromatid exchanges (SCEs) was used as an index of cytogenetic damage. Proliferation rate index (PRI), mitotic index (MI), average generation time (AGT) and population doubling time (PDT) were also evaluated. A significant $(p<0.01)$ increase in SCE levels along with a significant $(p<0.01)$ reduction in PRIs and MIs were observed in women with PCOS compared with healthy subjects. Additionally, subgroup $C$ demonstrated statistically significant differences compared with others, while subgroup A had similar results with healthy females. Our results suggest that females with PCOS show increased chromosomal instability in peripheral lymphocytes and a consequent inability of the cells to promote their own mitotic cycle. A positive correlation between DNA damage and PCOS phenotypes is also reported.

\section{Keywords}

Polycystic Ovary Syndrome, Proliferative Rate Index, Mitotic Index, Genotoxicity, Cytostaticity

\footnotetext{
${ }^{*}$ Corresponding author.
}

How to cite this paper: Dafa, E., et al. (2015) Enhancement of Sister Chromatid Exchanges (SCEs) in Peripheral Blood Lymphocytes of Women with Polycystic Ovary Syndrome (PCOS) in Vitro. Open Journal of Obstetrics and Gynecology, 5, 378384. http://dx.doi.org/10.4236/ojog.2015.57055 


\section{Introduction}

Polycystic ovary syndrome (PCOS) is one of the most common endocrine disorders amongst women of reproductive age. It is characterized by hyperandrogenism, menstrual disturbances, infertility due to chronic anovulation and polycystic ovaries [1]. Previous studies have demonstrated that the prevalence of the syndrome in different populations ranges from $2 \%-20 \%$, depending on which diagnostic criteria are used [2]-[4].

It is generally admitted that PCOS is a multifactorialdisorder that results from a combination of multiple gene polymorphisms under the influence of environmental factors [5]-[7]. Cytogenetic studies have shown that women with PCOS have increased damage in their genetic material [8]-[10]. Additionally, a relation between PCOS and $\mathrm{X}$ chromosome aneuploidy, including XX/XO mosaics and high proportion of X chromosome abnormal segregation has been reported in a limited number of studies [8]-[11].

Today, cytogenetics provides several biomarkers for chromosomal instability assessment, one of which is thesister chromatid exchange (SCE) frequency in cells. SCE is a natural process that implicates the exchange of homologous genetic segments as a mode of repair mechanism. The methodology of SCEs has been proved to be a very useful tool with predictive value, for detecting harmful effects on DNA, caused by various physical and chemical factors. Increased frequency of this index reflects the existence of genotoxicity in cells and the subsequent failure of repair mechanisms to recover the damaged site. Several studies have reported that SCE analysis is a very sensitive method, able to detect mutagens and/or carcinogens [12] and maybe more sensitive than other cytogenetic endpoints, such as micronuclei and chromosome aberrations [13]. Additionally, Proliferation Rate Index (PRI) and Mitotic Index (MI) are sensitive indices of cellular toxicity by antimutagenic and chemotherapeutic agents [14] [15].

The aim of the present study was the quantitative and qualitative evaluation of DNA damage on peripheral lymphocytes of females with PCOS through the SCE assay and the determination of a possible association between chromosomal instability and different phenotypes of the syndrome. In order to reveal any underlying chromosome instability, irinotecan (CPT-11), a known antineoplasticdrug, was used as a positive control to produce SCEs. CPT-11 acts as an inhibitor of topoisomerase I, an essential enzyme in the DNA replication [16], increasing chromosomal aberrations and reducing cell viability.

\section{Materials and Methods}

\subsection{Patients}

Heparinized blood samples were received from 22 females (average age 26.4 years) newly diagnosed with PCOS and 22 healthy women (average age 29.5 years) served as control subjects. PCOS was diagnosed according to the Rotterdam consensus criteria [17]. The patients were further divided into three subgroups according to the phenotypic characteristics. Subgroup A includes 4 patients with ovulatory dysfunction: oligo- and/or anovulation (ANOV) and polycystic ovaries (PCO), Subgroup B includes 6 patients who are positive in two of three criteria including hyperandrogenism (HA) (HA + ANOV or HA + PCO), while Subgroup C contains 12 patients with all three conditions present (HA + ANOV + PCO). All samples were obtained from the Department of Obstetrics and Gynaecology of Alexandroupolis University Hospital. All participants were fully informed and gave written consent. The study protocol was approved by the University General Hospital of Alexandroupolis Ethics Committee.

\subsection{Protocol}

Cultures of peripheral lymphocytes were prepared in universal containers by adding 11 drops of whole blood to $5 \mathrm{ml}$ of Chromosome Medium B (Biochrom KG, Berlin, Germany). At the beginning of the culture period, CPT-11 and 5-bromodeoxyuridine (BrdU) were added ata finalconcentration of $50 \mathrm{ng} / \mathrm{ml}$ and $5 \mu \mathrm{g} / \mathrm{ml}$, respectively. The cultures were incubated at $37^{\circ} \mathrm{C}$ for $72 \mathrm{~h}$. After $70 \mathrm{~h}, 0.5 \mu \mathrm{g} / \mathrm{ml}$ of Colcemide was added for additional incubation of $2 \mathrm{~h}$. Cell harvesting followed. The chromosomes were stained by a modified Fluorescence plus Giemsa (FPG) technique [14].

Three main indices were evaluated: a) SCEs, which is a qualitative and quantitative index of genotoxicity, b) the mitotic index (MI), which is a qualitative index of cytotoxicity and c) the proliferation rate index (PRI), which is a qualitative index of cytostaticity. In relation to cell cycle kinetics the Average generation time (AGT) and the Population doubling time (PDT) were also estimated. 
Mean SCE levels were evaluated only in suitable second division metaphases. 20 well spread and differentiated metaphases were counted per culture, because only at this stage were we able to observe and count them (Figure 1 and Figure 2). In order to establish PRI, 200 cells were counted and the following formula was used: $\mathrm{PRI}=(\mathrm{M} 1+2 \mathrm{M} 2+3 \mathrm{M} 3+) / \mathrm{N}$, where $\mathrm{M} 1$ is the percentage of cells at first division, $\mathrm{M} 2$ is the percentage of cells at second division and M3+ is the percentage of cells at third and subsequent divisions, while $\mathrm{N}$ is the total number of cells counted. For the MIs, 2000 activated lymphocytes were determined for each culture. The cell cycle time AGT was studied as the ratio of BrdU time and proliferation rate index, AGT $=72 \mathrm{~h}$ (BrdU time)/PRI. Finally, PDT is the time in which cells divide, i.e. in one cell cycle of $24 \mathrm{~h}$. The following formula was used for this index: PDT $=24 \mathrm{~h} / \mathrm{PRI}[18]$.

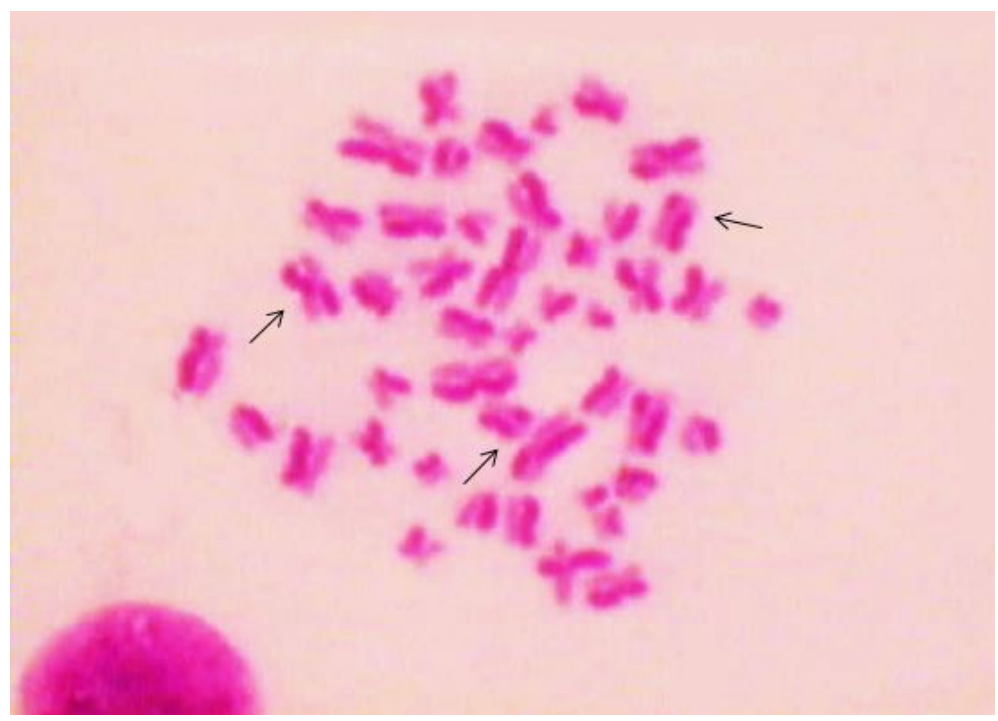

Figure 1. A second-division metaphase after Fluorescence Plus Giemsa staining of a healthy woman blood lymphocyte, where all chromosomes have one lightly stained chromatid and one heavily stained chromatid (arrows show sister chromatid exchanges). Original magnification 1000×.

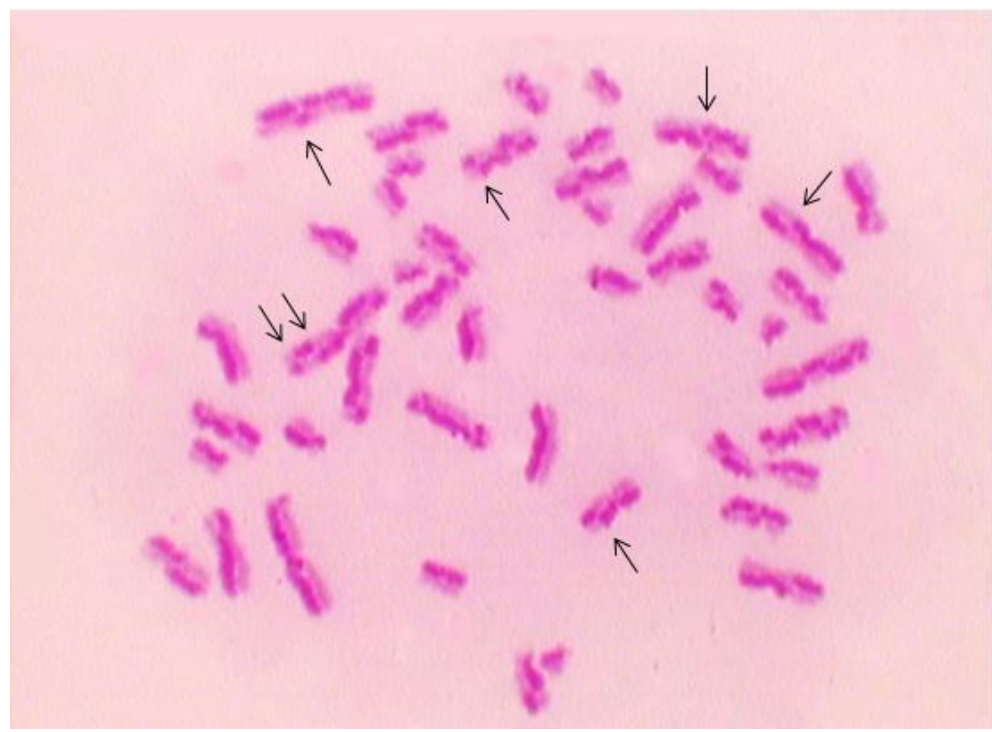

Figure 2. A second-division metaphase after Fluorescence Plus Giemsa staining of a PCOS blood lymphocyte, where all chromosomes have one lightly stained chromatid and one heavily stained chromatid (arrows show sister chromatid exchanges). Original magnification $1000 \times$. 


\subsection{Statistical Analysis}

One-way analysis of variance (ANOVA) was used to assess differences of SCE frequency between the two main groups and the subgroups of patients, while post hoc analysis was performed using Dunnett's T3 test for pair-wise comparisons. Statistical evaluation of PRI, MI AGT and PDT was based on Chi-squared test. A probability $\mathrm{p}<0.05$ was considered as a statistically significance.

\section{Results}

Table 1 illustrates our findings in control group, PCOS group and PCOS subgroups. The cytogenetic parameters were remarkably different between the two main groups. Women with PCOS demonstrated a significantly (p < 0.01 ) increased SCE frequency at a percentage of 19.9\%, having a mean value of 8.27 SCEs/cell compared with the control subjects having a mean value of $6.9 \mathrm{SCEs} / \mathrm{cell}$ in untreated cultures. The mutagenic agent CPT-11 gave rise to a significant increase in SCE levels in both groups. The values were estimated in $30.79 \mathrm{SCEs} / \mathrm{cell}$ and 35.05 SCEs/cell for controls and patients, respectively. Especially in PCOS patients, subgroup A had similar results with the control group. Subgroup B reveals statistically significant $(\mathrm{p}<0.01)$ induction of SCE levels (8.28 SCEs/cell) in control lymphocyte cultures (without CPT-11 treatment) in comparison with subgroup A (6.99 SCEs/cell) and control group, but not with subgroup C (8.7 SCEs/cell). Subgroup C indicates the strongest chromosomal instability, which is obvious from the statistically significant $(\mathrm{p}<0.01)$ induction of SCEs/cell with or without CPT administration.

As far as the mitotic index $(\mathrm{MI})$ is concerned, patients showed significant $(\mathrm{p}<0.01)$ reduction in the number of mitotic divisions (33.6\%) in comparison with healthy subjects (41.4\%). The cytotoxic activity of CPT-11 was exhibited in all cultures that were incubated. CPT-11 gave a significant decrease in the number of cell divisions at a percentage $16.96 \%$ in women with PCOS, but a greater reduction in healthy females at a percentage $18.84 \%$. However, the MI values in CPT-11 cultures were $27.9 \%$ in patients and $33.6 \%$ in healthy subjects. Furthermore, subgroup C had lower values of MI than the other subgroups. Subgroup B showed a significant decrease in MI compared with subgroup A. Moreover, healthy women and subgroup A had similar results.

Finally, Table 1 exhibits details of the cell-cycle progression in the two main groups and PCOS subgroups. PCOS women illustrated a significant $(\mathrm{p}<0.01)$ decrease in PRI and higher $(\mathrm{p}<0.01)$ AGT and PDT values compared with control group (exposed or not to CPT agent). Additionally, subgroup C demonstrated higher cytostaticity, as revealed by the PRI, AGT and PDT values, followed by subgroup B and lastly by subgroup A.

\section{Discussion}

The SCE frequency in cultured lymphocytes has been used as biomarker of chromosomal damages for many years. In this paper we reported for the first time SCE frequencies in peripheral blood lymphocytes of women

Table 1. Mean number of SCEs, mitotic index (MI) and proliferation rate index (PRI) in cultured peripheral lymphocytes of patients, healthy women and PCOS subgroups. Average generation time (AGT) and population doubling time (PDT) are also shown.

\begin{tabular}{|c|c|c|c|c|c|c|}
\hline Donors & Cultures & SCEs/cell \pm SEM & Mitotic index (\%o) & PRI & AGT & PDT \\
\hline \multirow{2}{*}{ Control group } & Control & $6.90 \pm 0.16$ & 41.4 & 2.56 & 28.27 & 9.42 \\
\hline & $C P T-11$ & $30.79 \pm 0.53^{\mathrm{a}}$ & $33.6^{\mathrm{a}}$ & $2.46^{\mathrm{a}}$ & $29.82^{\mathrm{a}}$ & $9.94^{\mathrm{a}}$ \\
\hline \multirow{2}{*}{ PCOS group } & Control & $8.27 \pm 0.17^{\mathbf{b}}$ & $33.6^{\mathbf{b}}$ & $2.42^{\mathrm{b}}$ & $29.93^{\mathbf{b}}$ & $9.98^{\mathrm{b}}$ \\
\hline & $C P T-11$ & $35.05 \pm 0.57^{\mathrm{ab}}$ & $27.9^{\mathbf{a b}}$ & $2.30^{\mathbf{a b}}$ & $31.42^{\mathrm{ab}}$ & $10.47^{\mathrm{ab}}$ \\
\hline \multirow{2}{*}{ Subgroup A } & Control & $6.99 \pm 0.35^{\mathrm{c}}$ & $39.5^{c}$ & $2.55^{\mathrm{c}}$ & $28.33^{c}$ & $9.44^{\mathrm{c}}$ \\
\hline & $C P T-11$ & $29.57 \pm 1.01^{\mathrm{ad}}$ & $34.4^{\mathrm{ac}}$ & $2.44^{\mathrm{ac}}$ & $29.27^{\text {ac }}$ & $9.76^{\mathrm{ac}}$ \\
\hline \multirow{2}{*}{ Subgroup B } & Control & $8.28 \pm 0.29^{e}$ & $36.2^{\mathbf{b c}}$ & $2.46^{\mathrm{bc}}$ & $29.50^{\mathrm{bc}}$ & $9.83^{\mathrm{bc}}$ \\
\hline & $C P T-11$ & $30.78 \pm 0.96^{\text {ad }}$ & $29.3^{\mathrm{abc}}$ & $2.29^{\text {af }}$ & $31.62^{\text {af }}$ & $10.54^{\text {af }}$ \\
\hline \multirow{2}{*}{ Subgroup C } & Control & $8.70 \pm 0.23^{\mathrm{e}}$ & $30.3^{\mathbf{b c}}$ & $2.27^{\mathbf{b c}}$ & $31.85^{\mathrm{bc}}$ & $10.62^{\mathrm{bc}}$ \\
\hline & $C P T-11$ & $39.01 \pm 0.79^{\mathbf{b c}}$ & $25.1^{\mathrm{abc}}$ & $2.25^{f}$ & $32.46^{\mathrm{f}}$ & $10.82^{f}$ \\
\hline
\end{tabular}

${ }^{\mathrm{a}} \mathrm{p}<0.01$ vs control culture; ${ }^{\mathrm{b}} \mathrm{p}<0.01$ vs control group; ${ }^{\mathrm{c}} \mathrm{p}<0.01$ vs other subgroups; ${ }_{\mathrm{p}}^{\mathrm{d}}<0.01$ vs subgroup $\mathrm{C}$; ${ }^{\mathrm{e}} \mathrm{p}<0.01$ vs control group and subgroup $\mathrm{A} ; \mathrm{p}<0.05$ vs control group and subgroup $\mathrm{A}$. 
with PCOS. Additionally, to the best of our knowledge this is the first study to investigate a possible association between chromosomal instability and clinical phenotypes of the syndrome. Our findings illustrated that the DNA of patients showed significant damage, as shown by the increased SCE frequency in lymphocytes, confirming some reports that deal with this issue [8] [19]. These reports suggest the presence of genetic abnormality in PCOS [9] [10] [20]. Whether the increased chromosomal instability in PCOS patients is a side effect of the disease process or is due to altered genetic background remains unclear. TheCPT-11 agentled to significant genotoxicity in both groups, but it elicited a higher increase in SCE levels in patients than in healthy individuals. The induction of SCEs depends on the agent activity, which is associated with a different mechanism of creation of SCEs and probably depends on the composition of genetic material [21]. Additionally, the high rate of DNA damage in PCOS patients suggests that the repair mechanisms are more "lenient" and/or insufficient to genetic changes. The malfunction and the consequently deficiency of DNA-repair mechanisms in PCOS women have been reported [22].

Data from the control cultures of the two examined groups revealed the reduced viability and the inability of patients' lymphocytes to promote their own mitotic cycle, which is demonstrated by the significant low PRI, MI, AGT and PDT values. The mutagenic agent CPT-11 had a higher cytostatic effect on patients than on healthy individuals. It is known that CPT-11 is involved in DNA replication and cell division, reducing cell viability [23]. However, it is worth pointing out that this factor caused a greater reduction ratio of MI in healthy women than in patients. A possible explanation for this observation could be the fact that healthy individuals whose genetic material had not undergone any crucial alterations were more responsive to cytotoxic agents.

Significant differences in cytogenetic indices emerged between the subgroups of patients. This study demonstrates a possible association of chromosomal instability and different PCOS phenotypes. More specifically, females with severe PCOS ( 3 of 3 criteria) had clearly higher genotoxicity, cytostaticity and cytotoxicity than the other phenotypes. Patients with ovulatory dysfunction (ANOV and PCO) demonstrated similar results with healthy individuals. This finding possibly reinforces the conviction of many scientists that this phenotype should not be taken into consideration as a PCOS case. Moreover, patients with HA and ovarian dysfunction (ANOV or PCO) demonstrated a significant increase in spontaneous SCE frequency and considerable reduction in the cell cycle progression, as exhibited by the decreased PRI and MI along with increased AGT and PDT in comparison with controls and subgroup A. The observation of increased chromosomal damage in phenotypes which have androgen excess as one of the main characteristics (B and C subgroups) reveals the potential role of HA in genetic instability. Indeed, positive correlation between free testosterone and DNA strand breakage has been reported in PCOS patients [24]. It is believed that testosterone and DHEAS may have pro-oxidant properties depending on their concentration [25] [26]. Therefore, these hormones may contribute to the induction of oxidative DNA damage in the form of strand breakage at high concentrations. Thus, it would not be too arbitrary to point out that the HA in PCOS is associated with chromosomal instability.

Genetic instability can have very serious consequences for PCOS patients. It has been proved that chromosomal abnormalities (structural, numerical) are associated with increased risk of cancer [27] and early miscarriages [28]. There are studies supporting the existence of these two phenomena in PCOS patients [29] [30]. It is generally accepted that PCOS is associated with gynaecological malignancies such as endometrial cancer and less with ovarian cancer. There is a weak relation between breast cancer and the syndrome [31]. It is worth pointing out that clinical investigators have shown that SCE levels are higher in patients with ovarian and breast cancer than healthy individuals [32] [33]. Therefore, the mutagenic process and the consequent cancer risk in these women probably can be clarified with SCEs.

\section{Conclusion}

In conclusion, in this study SCEs have been applied as a predictive assay of DNA damage risk and as a biomarker of mutagenicity. Spontaneous or baseline SCE frequencies in peripheral blood lymphocytes provide an additional diagnostic tool for identifying accumulated chromosomal damage occurring during the lifespan of the cells. Our results suggest that there is chromosomal instability in lymphocytes of PCOS patients, which is associated with phenotypic characteristics. Future studies should be conducted to confirm these findings.

\section{Acknowledgements}

We thank all the women for participating in this study. 


\section{Conflicts of Interest}

The authors declare that there are no conflicts of interest. The authors alone are responsible for the content and writing of the paper.

\section{References}

[1] Sirmans, S.M. and Pate, K.A. (2013) Epidemiology, Diagnosis, and Management of Polycystic Ovary Syndrome. Clinical Epidemiology, 6, 1-13. http://dx.doi.org/10.2147/CLEP.S37559

[2] Azziz, R., Woods, K.S., Reyna, R., Key, T.J., Knochenhauer, E.S. and Yildiz, B.O. (2004) The Prevalence and Features of the Polycystic Ovary Syndrome in an Unselected Population. Journal of Clinical Endocrinology \& Metabolism, 89, 2745-2749. http://dx.doi.org/10.1210/jc.2003-032046

[3] March, W.A., Moore, V.M., Willson, K.J., Phillips, D.I., Norman, R.J. and Davies, M.J. (2010) The Prevalence of Polycystic Ovary Syndrome in a Community Sample Assessed under Contrasting Diagnostic Criteria. Human Reproduction, 25, 544-551. http://dx.doi.org/10.1093/humrep/dep399

[4] Yildiz, B.O., Bozdag, G., Yapici, Z., Esinler, I. and Yarali, H. (2012) Prevalence, Phenotype and Cardiometabolic Risk of Polycystic Ovary Syndrome under Different Diagnostic Criteria. Human Reproduction, 27, 3067-3073. http://dx.doi.org/10.1093/humrep/des232

[5] Barber, T.M. and Franks, S. (2013) Genetics of Polycystic Ovary Syndrome. Frontiers of Hormone Research, 40, 28-39. http://dx.doi.org/10.1159/000341682

[6] Diamanti-Kandarakis, E., Kandarakis, H. and Legro, R.S. (2006) The Role of Genes and Environment in the Etiology of PCOS. Endocrine, 30, 19-26. http://dx.doi.org/10.1385/ENDO:30:1:19

[7] Franks, S., McCarthy, M.I. and Hardy, K. (2006) Development of Polycystic Ovary Syndrome: Involvement of Genetic and Environmental Factors. International Journal of Andrology, 29, 278-285. http://dx.doi.org/10.1111/j.1365-2605.2005.00623.x

[8] Moran, L.J., Noakes, M., Clifton, P.M., Norman, R.J. and Fenech, M.F. (2008) Genome Instability Is Increased in Lymphocytes of Women with Polycystic Ovary Syndrome and Is Correlated with Insulin Resistance. Mutation Research, 639, 55-63. http://dx.doi.org/10.1016/j.mrfmmm.2007.11.007

[9] Nersesyan, A., Martirosyan, A., Parsadanyan, G. and Zalinyan, G. (2006) Chromosomal Aberrations Level in Peripheral Blood Lymphocytes of Women with Polycystic Ovary Syndrome. Journal of the Balkan Union of Oncology, 11, 477-480.

[10] Yesilada, E., Sahin, I., Ozcan, H., Yildirim, I.H., Yologlu, S. and Taskapan, C. (2006) Increased Micronucleus Frequencies in Peripheral Blood Lymphocytes in Women with Polycystic Ovary Syndrome. European Journal of Endocrinology, 154, 563-568. http://dx.doi.org/10.1530/eje.1.02117

[11] Hague, W.M., Adams, J., Reeders, S.T. and Jacobs, H.S. (1989) 45 X Turner’s Syndrome in Association with Polycystic Ovaries. Case Report. British Journal of Obstetrics and Gynaecology, 96, 613-618. http://dx.doi.org/10.1111/j.1471-0528.1989.tb03264.x

[12] Wilson III, D.M. and Thompson, L.H. (2007) Molecular Mechanisms of Sister Chro-Matid Exchange. Mutation Research, 616, 11-23. http://dx.doi.org/10.1016/j.mrfmmm.2006.11.017

[13] Lasne, C., Gu, Z.W., Venegas, W. and Chouroulinkov, I. (1984) The in Vitro Micro-Nucleus Assay for Detection of Cytogenetic Effects Induced by Mutagen-Carcinogens: Comparison with the in Vitro Sister-Chromatid Exchange Assay. Mutation Research, 130, 273-282. http://dx.doi.org/10.1016/0165-1161(84)90103-1

[14] Maskaleris, T., Lialiaris, T. and Triantaphyllidis, C. (1998) Induction of Cytogenetic Damage in Human Lymphocytes in Vitro and of Antineoplastic Effects in Ehrlich Ascites Tumor Cells in Vivo Treated by Methotrexate, Hyperthermia and/or Caffeine. Mutation Research, 422, 229-236. http://dx.doi.org/10.1016/S0027-5107(98)00198-5

[15] Lialiaris, T., Mourelatos, D. and Dozi-Vassiliades, J. (1988) Enhancement of Cytoge-Netic Damage by Chlorpromazine in Human Lymphocytes Treated with Alkylating Antineoplastics and Caffeine. Mutation Research, 206, 361-365. http://dx.doi.org/10.1016/0165-1218(88)90122-X

[16] Mathijssen, R.H., Loos, W.J., Verweii, J. and Sparreboom, A. (2002) Pharmacology of Topoisomerase I Inhibitors Irinotecan (CPT-11) and Topotecan. Current Cancer Drug Targets, 2, 103-123. http://dx.doi.org/10.2174/1568009023333890

[17] Rotterdam ESHRE/ASRM-Sponsored PCOS Consensus Workshop Group (2004) Revised 2003 Consensus on Diagnostic Criteria and Long-Term Health Risks Related to Polycystic Ovary Syndrome (PCOS). Fertility Sterility, 81, $19-25$.

[18] Avani, G. and Rao, M.V. (2007) Genotoxic Effects in Human Lymphocytes Exposed to Arsenic and Vitamin A. Toxi- 
cology in Vitro, 21, 626-631. http://dx.doi.org/10.1016/j.tiv.2006.12.010

[19] Nersesyan, A. and Chobanyan, N. (2010) Micronuclei and Other Nuclear Anomalies Levels in Exfoliated Buccal Cells and DNA Damage in Leukocytes of Patients with Polycystic Ovary Syndrome. Journal of the Balkan Union of Oncology, 15, 337-339.

[20] Hamurcu, Z., Bayram, F., Kahriman, G., Dönmez-Altuntas, H. and Baskol, G. (2010) Micronucleus Frequency in Lymphocytes and 8-Hydroxydeoxyguanosine Level in Plasma of Women with Polycystic Ovary Syndrome. Gynecological Endocrinology, 26, 590-595. http://dx.doi.org/10.3109/09513591003632142

[21] Wenger, L.S. (1995) Chemical Induction of Sister Chromatid Exchange at Fragile Sites. Cancer Genetics and Cytogenetics, 85, 72-74. http://dx.doi.org/10.1016/0165-4608(95)00137-9

[22] Manikkumar, R., Roy, D.D., Krishnan, V. and Vijayakumar, T. (2013) Association of DNA Damage and Dyslipidemia with Polycystic Ovarian Syndrome. Journal of Medical \& Allied Sciences, 3, $15-21$.

[23] Kopjar, N., Zeljezić, D., Vrdoljak, A.L., Radić, B., Ramić, S., Milić, M., Gamulin, M., Pavlica, V. and Fucić, A. (2007) Irinotecan Toxicity to Human Blood Cells in Vitro: Relationship between Various Biomarkers. Basic \& Clinical Pharmacology \& Toxicology, 100, 403-413. http://dx.doi.org/10.1111/j.1742-7843.2007.00068.X

[24] Dinger, Y., Akcay, T., Erdem, T., IIkerSayqili, E. and Gundogdu, S. (2005) DNA Damage, DNA Susceptibility to Oxidation and Glutathione Level in Women with Poly-Cystic Ovary Syndrome. Scandinavian Journal of Clinical and Laboratory Investigation, 65, 721-728. http://dx.doi.org/10.1080/00365510500375263

[25] Gallo, M., Aragno, M., Gatto, V., Tamagno, E., Brignardello, E. and Manti, R. (1999) Protective Effect of Dehydroepiandrosterone against Lipid Peroxidation in Human Liver Cell Line. European Journal of Endocrinology, 141, 35-39. http://dx.doi.org/10.1530/eje.0.1410035

[26] Chainy, G.B.N., Samantaray, S. and Samanta, L. (1997) Testosterone Induced Changes in Testicular Antioxidant System. Andrologia, 29, 343-349. http://dx.doi.org/10.1111/j.1439-0272.1997.tb00328.x

[27] Migliore, L. and Coppede, F. (2002) Genetic and Environmental Factors in Cancer Neurodegenerative DISEASES. Mutation Research, 512, 135-153. http://dx.doi.org/10.1016/S1383-5742(02)00046-7

[28] Hassold, T., Chen, N., Funkhouser, J., Jooss, T., Manuel, B., Matsuura, J., Matsu-yama, A., Wilson, C., Yamane, J.A. and Jacobs, P.A. (1980) A Cytogenetic Study of 1000 Spontaneous Abortions. Annals of Humans Genetics, 44, 151178. http://dx.doi.org/10.1111/j.1469-1809.1980.tb00955.x

[29] Gadducci,A., Gargini, A., Palla, E., Fanucchi, A. and Genazzani, A.R. (2005) Poly-Cystic Ovary Syndrome and Gynecological Cancers: Is There a Link? Gynecological Endocrinology, 20, 200-208. http://dx.doi.org/10.1080/09513590400021201

[30] Sagle, M., Bishop, K., Ridley, N., Alexander, F.M., Michel, M., Bonney, R.C., Beard, R.W. and Franks, S. (1988) Recurrent Early Miscarriage and Polycystic Ovaries. British Medical Journal, 297, 1027-1028. http://dx.doi.org/10.1136/bmj.297.6655.1027

[31] Dumesic, D.A. and Lobo, R.A. (2013) Cancer Risk and PCOS. Steroids, 78, 782-785. http://dx.doi.org/10.1016/j.steroids.2013.04.004

[32] Baltaci, V., Kayikçioğlu, F., Alpas, I., Zeyneloğlu, H. and Haberal, A. (2002) Sister Chromatid Exchange Rate and Alkaline Comet Assay Scores in Patients with Ovarian Cancer. Gynecological Oncology, 84, 62-66. http://dx.doi.org/10.1006/gyno.2001.6450

[33] Dhar, P.K., Devi, S., Rao, T.R., Kumari, U., Joseph, A., Kumar, M.R., Nayak, S., Shreemati, Y., Bhat, S.M. and Bhat, K.R. (1996) Significance of Lymphocytic Sister Chromatid Exchange Frequencies in Ovarian Cancer Patients. Cancer Genetics and Cytogenetics, 89, 105-108. http://dx.doi.org/10.1016/0165-4608(95)00071-2 